\title{
Qualidade pós-produção de cultivares de gérbera de vaso fertirrigadas com soluções nutritivas ${ }^{(1)}$
}

\author{
FERNANDA LUDWIG(2), DIRCEU MAXIMINO FERNANDES(2,4), POLIANA ROCHA D'ALMEIDA MOTA ${ }^{(2)}$, ROBERTO LYRA VILLAS \\ BÔAS ${ }^{(2,4)}$ e DENISE LASCHI(5)
}

\begin{abstract}
RESUMO
O presente trabalho foi desenvolvido com o objetivo de avaliar a qualidade pós-produção de quatro cultivares de gérbera fertirrigadas com duas soluções nutritivas. O experimento foi realizado de maio a agosto de 2006, na Faculdade de Ciências Agrárias, da Universidade Estadual Paulista - Botucatu (SP). Utilizou-se o delineamento em blocos casualizados e constituído de um fatorial 4 x 2, sendo quatro cultivares de gérbera ('Cherry, Golden Yellow, Salmon Rose e Orange) e duas concentrações de solução nutritiva, 0,92 e $1,76 \mathrm{dS} \mathrm{m}^{-1}$ de condutividade elétrica (CE), na fase vegetativa e 1,07 e $2,04 \mathrm{dS} \mathrm{m}^{-1}$, na reprodutiva. O período experimental foi dividido em duas etapas, a primeira corresponde à produção, desenvolvida em ambiente protegido, e a segunda, ao período pós-produção, em laboratório. O enfoque do presente trabalho deu-se principalmente na segunda etapa. Aplicaram-se as nutritivas, durante a primeira fase, até que as plantas atingissem o ponto de comercialização, quando então foram levadas para laboratório com temperatura ambiente, no Departamento de Produção Vegetal - Horticultura, para avaliação pós-produção; logo após, iniciou-se a segunda etapa. A avaliação pós-produção foi desenvolvida no período de junho a agosto de 2006 - cada vaso permaneceu na sala por um período de 21 dias. Os vasos foram dispostos sobre bancadas, sem receber tratamento adicional. Avaliou-se o número de folhas e diâmetro do vaso no primeiro dia da pós-produção. Semanalmente, mediu-se o diâmetro de inflorescência e, no primeiro e último dia, a altura de planta. De acordo com os resultados obtidos, é possível concluir que os cultivares analisados têm sua qualidade mantida até os 14 dias, sendo esta reduzida drasticamente até os 21 dias de armazenamento. Desse modo, a comercialização desses cultivares, realizada nas mesmas condições do presente experimento, restringe-se a um curto período de tempo.

Palavras-chave: Gerbera jamesonii, nutrição de plantas, longevidade.
\end{abstract}

\section{ABSTRACT}

Post-production quality in potted gerbera cultivars fertigated with nutritive solutions.

The aim of the present work was to evaluate post production quality in four gerbera cultivars under two levels of fertigation. The experiment was carried out from May to August, 2006 at FCA/UNESP - Botucatu, SP. It was installed in randomized blocks and constituted of a 4 x 2 factorial scheme, being 4 gerbera cultivars (Cherry, Golden Yellow, Salmon Rose and Orange) and 2 concentration nutritive solution, 0,92 and 1,76 dS m${ }^{-1}$, during vegetative period and 1,07 e 2,04 dS m$~ m^{-1}$, during the reproductive one. The experimental period was divided in two stages, the first correspondent to the production, developed in greenhouse, and the second, correspondent the post-production, developed in laboratory. The present work is focused mainly in the second stage. The solutions were applied until the plants reach the marketing, when then they were taken for room with ambient temperature, in the DVP - Horticulture, for evaluation post-production, when the second stage was begun. The evaluation was developed in the period of June to August of 2007, however, each vase stayed at room for 21 days. The pots were disposed on benches, without additional treatment. Was evaluated the number of leaves, plant diameter, in the first day post-production. Weekly, the inflorescence diameter was measured, and in the first and last day (0 and 21), the plant height. The cultivars had your quality maintained until 14 days, being this reduced drastically until the 21 days of storage. The commercialization of these cultivars, in the same conditions of the present experiment, is limited to a short period of time.

Key-words: Gerbera jamesonii, plant nutrition, longevity.

(') Trabalho recebido para publicação em 20/12/2007 e aprovado em 04/08/2008.

( ${ }^{2}$ ) Departamento de Recursos Naturais / Ciência do Solo, Faculdade de Ciências Agrárias (FCA), Universidade Estadual Paulista (UNESP).

Fazenda Experimental Lageado, Caixa Postal 237, 18610-307 Botucatu (SP).

(3) Autor para correspondência: fludwig@fca.unesp.br

(4) Bolsista CNPq.

(5) Departamento de Produção Vegetal / Horticultura, FCA/UNESP. 


\section{INTRODUÇÃO}

A gérbera é uma planta herbácea, pertencente à família Asteraceae, com inflorescência terminal em capítulo. O comprimento do pedúnculo define seu uso, sendo longo para flor de corte e curto para vaso. O capítulo floral é formado, do exterior para o interior, por várias filas concêntricas de flores femininas liguladas, normalmente uma fila de flores hermafroditas não funcionais e, ao centro, as flores masculinas. As flores liguladas são de forma e espessura variadas e com ampla gama de cores, variando de acordo com os diferentes cultivares (INFOAGRO, 2005).

A comercialização da gérbera de corte na América do Norte teve início em 1920, entretanto, o primeiro cultivar selecionado para desenvolvimento em vaso foi introduzido no Japão, no início de 1980 (ROGERS e TJIA, 1990). No Brasil, a produção se concentra principalmente no Estado de São Paulo.

Rosa, violeta, branca, amarela e vermelha são as cores em ordem de preferência, e a crescente tendência na diversidade é apresentada em mais de 300 variedades lançadas a cada ano (CHUNG et al., 2001). Em função da variedade de cores, apresenta-se como cultura de elevado valor comercial, tanto para o mercado interno quanto para a exportação (MOTA, 2007), com destaque nos principais centros de comercialização.

As variações entre cultivares, no entanto, envolvem fatores além da coloração. De acordo com MOTA (2007), os cultivares 'Cherry', 'Golden Yellow' e 'Salmon Rose' apresentam variabilidade em relação à demanda por nutrientes, às características produtivas e ao ciclo de crescimento, ao avaliar o manejo da fertirrigação, com diferentes níveis de condutividade elétrica.

Segundo SONNEVELD et al. (1999), a gérbera é classificada como moderadamente sensível à condutividade elétrica da solução nutritiva, variando de acordo com o ambiente e o cultivar, sendo esses fatores importantes para a obtenção de informações sobre o manejo de soluções nutritivas com concentrações específicas. BELLÉ (1998) concorda com esta informação, reforçando que o desconhecimento dessa característica pode comprometer as práticas de irrigação e adubação, ocasionando desequilíbrios nutricionais na produtividade.

As características relacionadas com a qualidade são diretamente afetadas pelos fatores de cultivo. Segundo NELL et al. (1997) o potencial para a qualidade das flores de vaso e a máxima longevidade são determinados durante a produção, sendo afetados pelos cultivares, nutrição, práticas de irrigação, meio de crescimento e ambiente de cultivo. Segundo o mesmo autor, a nutrição é determinante na longevidade pós-produção. ROUDE et al. (1991) concordam que a prática da fertilização tem efeito significativo na longevidade de várias espécies de flores envasadas. $\mathrm{O}$ alto conteúdo de sais no substrato pode causar danos nas raízes e diminuir a manutenção da qualidade, com prematura senescência das flores e queda das folhas (TER HELL e HENDRICKS, 1995).

Durante a avaliação da qualidade do produto final deve-se levar em consideração a aparência da planta e as características internas, como longevidade, sendo determinada pela interação entre o potencial genético e as condições de cultivo (NOORDEGRAAF, 1994). Desse modo, o presente trabalho foi desenvolvido com o objetivo de avaliar a longevidade pós-produção de quatro cultivares de gérbera de vaso, analisadas com duas concentrações de solução nutritiva.

\section{MATERIAL E MÉTODOS}

O presente trabalho foi desenvolvido no Departamento de Recursos Naturais - Área de Ciência do Solo da Faculdade de Ciências Agrárias, da Universidade Estadual Paulista (UNESP), município de Botucatu, SP, com coordenadas geográficas de $21^{\circ} 51^{\prime}$ de latitude sul e $48^{\circ} 26^{\prime}$ de longitude oeste, no período de maio a agosto de 2006 .

O período experimental foi dividido em duas etapas, a primeira corresponde à fase de produção das plantas em casa de vegetação e a segunda, à fase de pósprodução. O presente trabalho tem o intuito de analisar as características de qualidade das plantas durante a segunda etapa experimental.

Vasos de gérbera foram observados durante a primeira fase experimental em casa de vegetação com estrutura de teto em arco, com laterais de tela branca, pavimentada com concreto, em uma área total de $168 \mathrm{~m}^{2}$ ( 7 × $24 \mathrm{~m})$ e 2,6 $\mathrm{m}$ de pé-direito. Objetivando o estudo da cultura numa intensidade luminosa (Lux) inferior a 50.000 lux, instalou-se na parte superior da estrutura, uma malha termorefletora com $50 \%$ (Aluminet ${ }^{\circledR}$ ), a qual permanecia fechada das $10 \mathrm{~h} 30 \mathrm{~min}$ às $16 \mathrm{~h}$ e aberta nos demais horários. A temperatura média no interior da casa de vegetação foi de $20,5^{\circ} \mathrm{C}$ e a umidade relativa média do ar de $69 \%$.

Utilizaram-se no experimento mudas de gérbera (Gerbera jamesonii), para cultivo em vaso, as quais foram previamente aclimatadas, por um período de 30 dias, e plantadas em vasos número $15(1,3 \mathrm{~L})$, preenchidos com substrato composto de $70 \%$ de casca de pinus fina e $30 \%$ de terra de subsolo.

O delineamento experimental adotado foi o de blocos casualizados, empregando-se o esquema fatorial $4 \times 2$ (4 cultivares e 2 soluções nutritivas), com 5 repetições. Os cultivares utilizados são provenientes da geração $F_{1}$ do grupo Festival, da empresa Sakata ${ }^{\circledR}$, sendo 'Cherry' e 'Golden Yellow', pertencentes à série Dark Eyes (centro escuro), e 'Salmon Rose' e 'Orange', pertencentes à série Light Eyes (centro claro).

As soluções nutritivas constituíram-se de duas condutividades elétricas (CE), sendo a primeira de 1,76 $\mathrm{dS} \mathrm{m} \mathrm{m}^{-1}(\mathrm{~S} 2)$ com a seguinte composição em $\mathrm{mg} \mathrm{dm}^{-3}$ : $142,0 \mathrm{NO}_{3}^{-} ; 101,5 \mathrm{NH}_{4}^{+} ; 105,0 \mathrm{~K} ; 25,2 \mathrm{P} ; 51,3 \mathrm{Ca} ; 6,3$ $\mathrm{Mg} ; \quad 28,0 \quad \mathrm{~S} ; \quad 0,2 \quad \mathrm{~B} ; \quad 0,3 \quad \mathrm{Cu} ; \quad 3,9 \mathrm{Fe}$; 1,4 Mn; 0,1 Mo e 0,3 Zn, no período vegetativo. A solução com CE de $0,92 \mathrm{dS}^{-1}(\mathrm{~S} 1)$ apresentava metade da concentração de sais. As soluções foram reformuladas quando a planta entrou na fase reprodutiva, aos 41 dias após a aclimatação (DAA), em razão da mudança na demanda da cultura, tendo uma CE de $2,04 \mathrm{dS} \mathrm{m}^{-1}$ (S2) e a seguinte composição em mg dm${ }^{-3}: 110,3 \mathrm{NO}_{3}^{-}, 66,8$ $\mathrm{NH}_{4}^{+} ; 285,0 \mathrm{~K} ; 56,6 \mathrm{P} ; 26,3 \mathrm{Ca} ; 17,18 \mathrm{Mg}$; 
$76 \mathrm{~S} ; 0,4 \mathrm{~B} ; 0,4 \mathrm{Cu} ; 4,4 \mathrm{Fe} ; 1,7 \mathrm{Mn} ; 0,1 \mathrm{Mo}$ e 0,5 Zn. A exemplo do que ocorreu na fase vegetativa, a solução com $\mathrm{CE}$ de $1,07 \mathrm{dS} \mathrm{m}^{-1}$ (S1) apresentou metade da concentração de sais.

As plantas receberam os nutrientes via fertirrigação, aplicados uma vez ao dia, sendo acompanhado os valores de $\mathrm{CE}$ e pH da solução do substrato, semanalmente, entretanto, sem realizar o manejo da solução nutritiva aplicada, a qual permaneceu com $\mathrm{CE}$ constante ao longo do ciclo. A lâmina de irrigação correspondeu à quantidade de água requerida, a fim de elevar a umidade do substrato contido no vaso ao valor correspondente à condição de máxima retenção (FANELA et al., 2006).

A segunda fase experimental foi desenvolvida no período de 30 de junho a 2 de agosto de 2006. Definiu-se como critério para estabelecer o início do período da pósprodução, o ponto de comercialização (PC), definido por LIN e FRENCH (1985) como o momento em que ocorre a abertura de dois ou mais círculos de estames, com liberação de pólen.

Em virtude da variabilidade dos tratamentos em relação ao $\mathrm{PC}$, os vasos que eram destinados a essa avaliação (1 por parcela) foram monitorados nas segundas, quartas e sextas-feiras e levados ao laboratório. Desse modo, seguiram-se as datas de 58,61, 63, 65, 68 e 70 DAA para o monitoramento do ponto de comercialização. O cultivar 'Orange' teve seu ponto de comercialização anterior aos demais, seguido de 'Golden Yellow', 'Salmon Rose' e 'Cherry'.

Os vasos permaneceram por 21 dias, em temperatura ambiente, no laboratório do Departamento de Produção Vegetal - Horticultura, UNESP. Dispostos sobre bancadas sem receber tratamento adicional, foram irrigados apenas com água de torneira a cada 2 dias, com $50 \mathrm{~mL} \mathrm{vaso}^{-1}$. Durante o período pós-produção, as janelas da sala permaneciam abertas das $8 \mathrm{~h}$ às $17 \mathrm{~h} 30 \mathrm{~min}$.

Sendo a qualidade influenciada pelas características vegetativas e reprodutivas, avaliou-se o número de folhas, o diâmetro foliar do vaso, o número e o diâmetro das inflorescências e a altura da planta.

No primeiro dia de avaliação, contabilizou-se o número total de folhas por planta, bem como a determinação do diâmetro foliar, para cada parcela, adotando duas extremidades da superfície foliar, perpendiculares entre si, com régua graduada em milímetro.

Semanalmente, aos 0, 7, 14 e 21 dias, avaliou-se o diâmetro de inflorescência para cada parcela, tomando-se duas medidas em pontos extremos, perpendiculares entre si. Todas as inflorescências abertas eram medidas, e os resultados foram expressos em valor médio, em milímetros. Essa avaliação foi efetuada com auxílio de paquímetro digital da marca Starrett, modelo 727. Contabilizou-se, também, o número total de inflorescências por planta.

No primeiro e último dia do experimento (dias $0 \mathrm{e}$ 21), realizou-se a avaliação da altura de planta, padronizando-se para isso, a altura da maior haste, efetuada com régua graduada em milímetro.

Os dados foram submetidos à análise da variância pelo teste F. As médias foram comparadas pelo teste de Tukey a $5 \%$ de probabilidade, quando os valores se mostraram significativos, com uso do programa estatístico Sisvar (FERREIRA, 2000). Procedeu-se ao desdobramento da interação entre cultivares e soluções nutritivas quando a análise da variância revelou efeito significativo.

\section{RESULTADOS E DISCUSSÃO}

A maior altura de planta foi constatada em 'Salmon Rose' e a menor em 'Orange', no primeiro dia de avaliação (tabela 1). Diferenças de altura entre cultivares de gérbera foram registradas em diversos trabalhos (SYROS et al., 2001; GOLDSBERRY e LANG, 1987 e SINGH e MANDHAR, 2001). Essa variável praticamente se manteve no final da avaliação, com pequenas variações, não sendo possível verificar diferença entre os cultivares.

As soluções nutritivas não promoveram efeito diferenciado na altura da planta, apesar de ocorrer tombamento de algumas hastes, principalmente em plantas fertirrigadas com a $\mathrm{S} 1$, o que ocorreu em torno de $60 \%$ destas. A falta de significância para essa variável, deve-se provavelmente ao fato de que a altura era aferida a partir do ponto mais extremo da planta.

SAVVAS et al. (2002) verificaram redução na altura de hastes de gérbera, em altos níveis de condutividade elétrica $\left(3,2 \mathrm{dS} \mathrm{m}^{-1}\right)$, superior aos que foram utilizados neste experimento. De modo semelhante, porém em plantas de crisântemo, JOINER e POOLE (1967) e STRINGHETA (1995) observaram redução no tamanho de plantas com o aumento dos sais solúveis no ambiente da raiz. Esses resultados, entretanto, diferem do observado neste trabalho, possivelmente pelo fato de o teor de sais fornecido pela S1 ser insuficiente para garantir estabilidade das hastes florais.

Outro fator com relevância e que deve ser considerado refere-se à demanda específica de nutrientes dos diferentes cultivares, variável também de acordo com a fase de desenvolvimento, observação esta constatada por MOTA (2007) em estudo com gérbera de vaso.

O cultivar 'Cherry' apresentou maior número de folhas $(26,8)$, sem diferir significativamente da Salmon Rose $(24,8)$. Quando as plantas foram manejadas com a $\mathrm{S} 1$, verificou-se de forma significativa um maior número de folhas (tabela 1). MOTA et al. (2007) ao estudarem o cultivar 'Cherry', encontraram um valor máximo de 21 folhas por planta, na condutividade elétrica de $2,0 \mathrm{dS} \mathrm{m}^{-1}$, ao final do ciclo de cultivo - superior ao registrado na $\mathrm{CE}$ de $1,0 \mathrm{dS} \mathrm{m}^{-1}$, sendo, desse modo, discordante os valores encontrados.

O diâmetro foliar do vaso não diferiu significativamente entre os cultivares e entre as soluções nutritivas (tabela 1). Entretanto, MOTA et al. (2007) observaram maiores diâmetros na $\mathrm{CE}$ de 2,0 $\mathrm{dS} \mathrm{m}^{-1}$, em relação à $\mathrm{CE}$ de $1,0 \mathrm{dS} \mathrm{m}^{-1}$. Os valores obtidos ( $33 \mathrm{~cm}$ em média) foram superiores aos encontrados por GUISELINI (2002), em gérbera de vaso $(22,6 \mathrm{~cm})$, e inferiores ao obtido por FANELA et al. (2006) para o cultivar 'Cherry' (46 cm). O diâmetro foliar tem grande importância em flores envasadas, para formação de um conjunto harmônico entre a parte vegetativa e as inflorescências, podendo ser considerado um indicativo da porção verde que compõe o vaso. 
De maneira geral, os cultivares 'Cherry' e 'Salmon Rose' apresentaram diâmetro de inflorescência superior, em grande parte das datas analisadas (tabela 2). GOLDSBERRY e LANG (1987), também verificaram que o diâmetro da inflorescência é variável de acordo com o cultivar. Obteve-se um coeficiente de correlação negativo entre o diâmetro e o número de inflorescência, para o cultivar 'Salmon Rose', indicando que o maior diâmetro é acompanhado pelo menor número de inflorescência $\left(\mathrm{r}^{2}\right.$ : $-0,84,-0,64$ e $-0,55$, para os dias 0,7 e 14 respectivamente).

Segundo NOORDEGRAAF (1994), o diâmetro de inflorescência é um indicativo de qualidade externa de flores. Os cultivares tiveram redução nessa variável ao final do período de análise, em ambas as soluções (Figura 1), em especial pela desidratação e abscisão das pétalas. Este decréscimo torna-se evidente ao analisar as equações de regressão apresentadas na tabela 3 .

Os menores valores de diâmetro de inflorescência foram registrados para o cultivar 'Golden Yellow', na S1 e para 'Orange' na S2. A redução dessa variável foi mais acentuada para a cultivar 'Salmon Rose' (figura 1).

$\mathrm{O}$ número de inflorescências não apresentou diferenças significativas entre cultivares e entre soluções nutritivas (Tabela 2), porém é possível verificar que os cultivares apresentaram comportamento semelhante entre si na $\mathrm{S} 2$, tanto para o número de inflorescência, quanto para o diâmetro. Observou-se, entretanto, aumento no número destas, principalmente até os 14 dias (figura 1). $\mathrm{Na} \mathrm{S} 1$ constatou-se uma amplitude maior entre os dados, indicando que os cultivares em solução com menor concentração de nutrientes possivelmente ajustaram-se de maneira diferenciada.

A durabilidade dos cultivares, indicada pelas variáveis analisadas, manteve-se idêntica nas duas soluções nutritivas até os 14 dias de armazenamento, concordando com SONNEVELD et al. (1999), os quais observaram que a vida de vaso de gérbera não é afetada pela salinidade. De acordo com SARZI et al. (2005), a suspensão da fertirrigação, nas duas últimas semanas, proporcionou maior longevidade em plantas de crisântemo. NELL et al. (1997) constataram que alguns cultivares de crisântemo têm maior longevidade quando a fertilização é terminada três semanas antes do ponto de comercialização, enquanto outros diminuem sua longevidade nessas condições. Isso indica que alguns cultivares são favorecidos positivamente com a redução da salinidade no meio de cultivo.

A estabilidade da cor dentro de ambientes internos é uma característica importante de qualidade (NOORDEGRAAF, 1994). Observou-se que a cultivar 'Salmon Rose' apresentou redução de intensidade na cor das pétalas nas novas inflorescências formadas durante $o$ período experimental, em $70 \%$ dos vasos, em ambas as soluções. Os cultivares chegaram aos 21 dias sem padrão de comercialização, com a totalidade das plantas apresentando injúrias como queimadura das pétalas, desidratação e abscisão das pétalas.

A manutenção da qualidade, avaliada pelo diâmetro de inflorescências, que expressa sua turgidez, foi mantida durante os 14 primeiros dias pós-produção. A partir desta data, constatou-se redução da qualidade e perda de valor comercial, pela presença de injúrias como queimadura, desidratação e abscisão das pétalas. Esse é um indicativo importante no que se refere à comercialização da gérbera envasada, analisada nas mesmas condições do experimento, pois o período para comercialização restringe-se a um curto espaço de tempo.

\section{CONCLUSÃo}

Com base nos resultados obtidos e nas condições em que o experimento foi desenvolvido, pode-se concluir que os cultivares utilizados têm sua qualidade pósprodução mantida até 14 dias de armazenamento, independentemente da solução nutritiva utilizada.

\section{AGRADECIMENTOS}

Os autores agradecem às empresas Steltenpool, Sakata e Ball Van Zanten, pela contribuição indispensável no desenvolvimento da pesquisa.

\section{REFERÊNCIAS}

BELLÉ, S. Sistemas de irrigação e concentrações de adubação complementar na produção de Gerbera jamesonii cv 1187 em vaso. 1998. 122f. Tese (Doutorado em Fitotecnia) - Universidade Federal do Rio Grande do Sul, Porto Alegre, 1998.

CHUNG, Y.M.; KIM, H.A.; KIM, K.Y.; PARK, S.W.; YI, Y.B.; LEE, J.H.; KNOW, O.C. Morphological characteristics and genetic variation of gerbera (Gerbera hybrida Hort.). Journal of Plant Biotechnology, FALTA O LOCAL, v.3, n.3, p.145-149, 2001.

FANELA, T.L.M.; MOTA, P.R.D.; VILLAS BÖAS, R.L.; LUDWIG, F.; FERNANDES, D.M. Influência de diferentes níveis de tensão de água na cultura de gérbera desenvolvida em substrato. In: $2^{\mathrm{a}}$ MOSTRA CIENTÍFICA EM CIÊNCIAS AGRÁRIAS, $10^{\mathrm{a}}$ MOSTRA CIENTÍFIA DA FMVZ E $13^{\mathrm{a}}$ REUNIÃO CIENTÍFICA EM CIÊNCIAS AGRÁRIAS DO LAGEADO. Resumos... Botucatu, 2006.

FERREIRA, D.F. Análises estatísticas por meio do Sisvar para Windows versão 4.0. In: 45ª Reunião Anual da Região Brasileira da Sociedade internacional de Biometria. Anais... UFSCar, São Carlos, SP, Julho de 2000. p.255-258.

GUISELINI, C. Microclima e produção de gérbera em ambientes protegidos com diferentes tipos de cobertura. Piracicaba: Escola Superior de Agricultura Luiz de Queiroz, Universidade de São Paulo, ESALQ/ USP, 2002, 53p. Dissertação (Mestrado Agronomia).

GOLDSBERRY, K.L.; LANG, R.C. Response of gerbera to root zone heating in soil and gravel substrates. HortScience, FALTA O LOCAL, v.22, n. 4, p. 595-597, 1987.

INFOAGRO. El cultivo de la gerbera. Disponível em: <http://www. infoagro.com/flores/flores/gerbera.htm>. Acesso em: jun. 2005. 
JOINER, J.N.; POOLE, R.T. Relationship of fertilization frequency to chrysanthemum yield and nutrient levels in soils and foliage. Proceedings of the American Society for Horticultural Science, Alexandria, v.90, p.397-402, 1967.

LIN, W.C.; FRENCH, C.J. Effects of supplementary lighting and soil warming on flowering of three gerbera cultivars. HortScience, Alexandria, v.20, n.2, p.271-273, 1985.

MOTA, P.R.D. Aplicação via fertirrigação de soluções com diferentes condutividades elétricas para produção de gérbera (Gerbera jamesonii L.) sob ambiente protegido. 2007. 149f. Tese (Doutorado em Agronomia/ Irrigação e Drenagem) - Universidade Estadual Paulista, Faculdade de Ciências Agronômicas, Botucatu, 2007.

MOTA, P.R.D.; VILLAS BÔAS, R.L.; LUDWIG, F.; FERNANDES, D.M. Crescimento da gérbera e produção de fitomassa seca em função de níveis de condutividade elétrica. In: $16^{\circ}$ Congresso Brasileiro de Floricultura e Plantas Ornamentais $/ 3^{\circ}$ Congresso Brasileiro de Cultura de Tecidos de Plantas / $1^{\circ}$ Simpósio de Plantas Ornamentais Nativas. Anais... Revista Brasileira de Floricultura e Plantas Ornamentais, p.1664-1667, 2007.

NELL, T.A.; BARRETT, J.E.; LEONARD, R.T. Production factors affecting postproduction quality of flowering potted plants. HortScience, Alexandria, v.32, n.5, p.817-819, 1997.

NOORDEGRAAF, C.V. Production and marketing of high quality plants. Acta Horticulturae, Wageningen, n.353, p.134-147, 1994.

ROGERS, M.N.; TJIA, B.O. Gerbera production. Timber Press Growers handbook series, v.4, 1990. 116p.
ROUDE, N.; NELL, T.A.; BARRETT, J.E. Nitrogen source and concentration, growin medium, and cultivar affect longevity of potted chrysantemums. HortScience, FALTA O LOCAL, v.26, n.1, p.49-51, 1991.

SARZI, I.; MOTA, P.R.D.A.; VILLAS BÔAS, R.L. Características químicas e longevidade de plantas envasadas de crisântemo em função da adubação final. Científica, Jaboticabal, v.33, n.1, p.57-61, 2005.

SAVVAS, D.; MANOS, G.; KOTSIRAS, A.; SOUVALIOTIS, S. Effects of silicon and nutrient induced salinity on yield flower quality and nutrient uptake of gerbera qrown in a closed hydroponic system. Journal of Applied Botany, v.76, n.5-6, p.153-158, 2002.

SINGH, K.P.; MANDHAR, S.C. Performance of exotic cultivars ofgerbera (Gerberajamesonii) under low costnaturallyventilated greenhouse environment. Indian Journal of Agricultural Sciences, FALTA O LOCAL, v.71, n.4, p.244-248, 2001.

SONNEVELD, C.; BAAS, R.; NIJSSEN, H.M.C.; HOOG, $\mathrm{J}$. Salt tolerance of flower crops grown in soilless culture. Journal of Plant Nutrition, v.22, n.6, p.1033-1048, 1999.

STRINGHETA, A.C.O. Avaliação de variedades de crisântemo em vaso, em substratos contendo composto de lixo urbano. Viçosa: Universidade Federal de Viçosa, UFV, 1995. 72p. Dissertação (Mestrado em Fitotecnia).

SYROS, T.; ECONOMOU, A.; EXARCHOU, E.; SCHMIDT, U. Flower and growth evalution of gerbera cultivated on perlite in an open hydroponic system. Acta Horticulturae, Wageningen, v.548, p.625-630, 2001.

TER HELL, B.; HENDRIKS, L. The influence of nitrogen nutrition on keeping quality of pot plants. Acta Horticulturae, Wageningen, v.405, p.138-147, 1995. 
Tabela 1. Valores médios de altura de planta, número de folhas e diâmetro foliar de vaso de quatro cultivares de gérbera de vaso fertirrigadas com duas soluções nutritivas. Botucatu (SP), 2006

Table 1. Mean values of plant height, number of leaves and vase diameter of four cultivar potted gerbera in two nutrient solutions. Botucatu (SP), 2006

\begin{tabular}{|c|c|c|c|c|}
\hline & \multicolumn{2}{|c|}{ Altura } & $\begin{array}{l}\text { Número de } \\
\text { folhas }\end{array}$ & Diâmetro foliar de vaso \\
\hline & \multicolumn{4}{|c|}{ Dias de análise } \\
\hline & 0 & 21 & 0 & 0 \\
\hline Cultivar & \multicolumn{3}{|c|}{----------------cm----------- } & -------cm------ \\
\hline Cherry & $29,8 \mathrm{ab}$ & $28,3 \mathrm{a}$ & $26,8 \mathrm{a}$ & $34,90 \mathrm{a}$ \\
\hline Golden Yellow & $30,0 \mathrm{ab}$ & $29,3 \mathrm{a}$ & $21,0 \mathrm{~b}$ & $31,13 \mathrm{a}$ \\
\hline Salmon Rose & $32,9 \mathrm{a}$ & 30,4 a & $24,8 \mathrm{ab}$ & $34,18 \mathrm{a}$ \\
\hline Orange & $28,2 \mathrm{~b}$ & $30,1 \mathrm{a}$ & $21,0 \mathrm{~b}$ & $31,83 \mathrm{a}$ \\
\hline \multicolumn{5}{|l|}{ Solução } \\
\hline $\mathrm{S} 1$ & $30,7 \mathrm{~A}$ & $27,9 \mathrm{~A}$ & $25,2 \mathrm{~A}$ & $32,89 \mathrm{~A}$ \\
\hline $\mathrm{S} 2$ & $29,7 \mathrm{~A}$ & $31,0 \mathrm{~A}$ & $21,5 \mathrm{~B}$ & $33,13 \mathrm{~A}$ \\
\hline $\mathrm{Cv}$ & $*$ & NS & $*$ & NS \\
\hline Sol & NS & NS & $*$ & NS \\
\hline $\mathrm{Cv} *$ Sol & NS & NS & NS & NS \\
\hline CV (\%) & 9,55 & 9,84 & 19,95 & 11,05 \\
\hline
\end{tabular}

Valores seguidos pela mesma letra na coluna não diferem entre si pelo teste de Tukey a $5 \%$. CV * Sol: interação entre cultivares e soluções nutritivas. NS: não significativo ao nível de $5 \%$. *: significativo a $5 \%$ de probabilidade. ${ }^{* *}$ : significativo a $1 \%$ de probabilidade. S1: CE de 0,92 e 1,07 dS m $\mathrm{m}^{-1}$; $2: 1,76$ e 2,04 $\mathrm{dS} \mathrm{m} \mathrm{m}^{-1}$ (ambos no período vegetativo e reprodutivo respectivamente). 
Tabela 2. Valores médios de diâmetro e número inflorescência de quatro cultivares de gérbera de vaso fertirrigadas com duas soluções nutritivas. Botucatu (SP), 2006

Table 2. Mean values offlower diameter and number of four cultivar potted gerbera in two nutrient solutions. Botucatu $(S P), 2006$

\begin{tabular}{|c|c|c|c|c|c|c|c|c|}
\hline & \multicolumn{4}{|c|}{ Diâmetro de inflorescência } & \multicolumn{4}{|c|}{ Número de inflorescências } \\
\hline & \multicolumn{8}{|c|}{ Dias de análise } \\
\hline & 0 & 7 & 14 & 21 & 0 & 7 & 14 & 21 \\
\hline Cultivar & \multicolumn{8}{|c|}{ 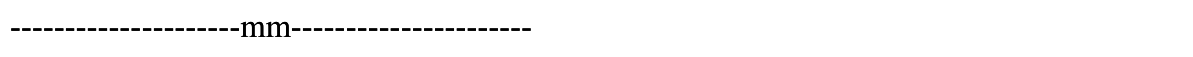 } \\
\hline Cherry & $92,8 \mathrm{ab}$ & $97,5 \mathrm{ab}$ & $93,3 \mathrm{a}$ & $86,1 \mathrm{a}$ & $3,2 \mathrm{a}$ & $4,1 \mathrm{a}$ & $4,4 \mathrm{a}$ & $4,2 \mathrm{a}$ \\
\hline Golden Yellow & $87,7 \mathrm{~b}$ & 87,7 b & $81,1 \mathrm{~b}$ & $71,1 \mathrm{~b}$ & $3,0 \mathrm{a}$ & $4,1 \mathrm{a}$ & $4,8 \mathrm{a}$ & $4,9 \mathrm{a}$ \\
\hline Salmon Rose & $103,1 \mathrm{a}$ & $104,2 \mathrm{a}$ & $94,2 \mathrm{a}$ & $71,8 \mathrm{~b}$ & $2,4 \mathrm{a}$ & $2,9 \mathrm{a}$ & $3,7 \mathrm{a}$ & $3,5 \mathrm{a}$ \\
\hline Orange & $90,0 \mathrm{~b}$ & $91,4 a b$ & $83,6 \mathrm{ab}$ & $73,5 \mathrm{~b}$ & $3,0 \mathrm{a}$ & $3,4 \mathrm{a}$ & $4,0 \mathrm{a}$ & $4,1 \mathrm{a}$ \\
\hline \multicolumn{9}{|l|}{ Solução } \\
\hline $\mathrm{S} 1$ & $96,3 \mathrm{~A}$ & $97,2 \mathrm{~A}$ & $89,3 \mathrm{~A}$ & $77,3 \mathrm{~A}$ & $2,8 \mathrm{~A}$ & $3,8 \mathrm{~A}$ & $4,4 \mathrm{~A}$ & $4,5 \mathrm{~A}$ \\
\hline $\mathrm{S} 2$ & $91,5 \mathrm{~A}$ & $93,2 \mathrm{~A}$ & $86,8 \mathrm{~A}$ & $74,0 \mathrm{~A}$ & $3,1 \mathrm{~A}$ & $3,5 \mathrm{~A}$ & $4,0 \mathrm{~A}$ & $3,8 \mathrm{~A}$ \\
\hline $\mathrm{Cv}$ & $*$ & $*$ & $*$ & $*$ & NS & NS & NS & NS \\
\hline Sol & NS & NS & NS & NS & NS & NS & NS & NS \\
\hline $\mathrm{Cv} * \mathrm{Sol}$ & NS & NS & NS & NS & NS & NS & NS & NS \\
\hline $\mathrm{CV}$ & 11,42 & 12,17 & 11,06 & 14,02 & 42,93 & 37,29 & 32,18 & 36,56 \\
\hline
\end{tabular}

Valores seguidos pela mesma letra na coluna não diferem entre si pelo teste de Tukey a $5 \%$. CV * Sol: interação entre cultivares e soluções nutritivas. NS: não significativo ao nível de $5 \%$. *: significativo a $5 \%$ de probabilidade. ${ }^{* *}$ : significativo a $1 \%$ de probabilidade. S1: CE de 0,92 e 1,07 dS m ${ }^{1}$; S2: 1,76 e 2,04

$\mathrm{dS} \mathrm{m}^{-1}$ (ambos no período vegetativo e reprodutivo respectivamente).

Tabela 3. Equações de regressão para o diâmetro de inflorescência de quatro cultivares de gérbera de vaso fertirrigadas com duas soluções nutritivas. Botucatu (SP), 2006

Table 3. Regression equations for flowers diameter of four cultivar potted gerbera in two nutrient solutions. Botucatu (SP), 2006

\begin{tabular}{lll}
\hline S1 & Equações de regressão & \\
\hline Cherry & $\mathrm{y}=-0,076 \mathrm{x}^{2}+1,087 \mathrm{x}+96,47$ & $\mathrm{R}^{2}=0,99$ \\
Golden Yellow & $\mathrm{y}=-0,018 \mathrm{x}^{2}-0,520 \mathrm{x}+86,69$ & $\mathrm{R}^{2}=0,99$ \\
Salmon Rose & $\mathrm{y}=-0,102 \mathrm{x}^{2}+0,853 \mathrm{x}+106,62$ & $\mathrm{R}^{2}=0,99$ \\
Orange & $\mathrm{y}=-0,057 \mathrm{x}^{2}+0,123 \mathrm{x}+98,33$ & $\mathrm{R}^{2}=0,97$ \\
\hline S2 & $\mathrm{y}=-0,044 \mathrm{x}^{2}+0,754 \mathrm{x}+89,76$ & $\mathrm{R}^{2}=0,88$ \\
\hline Cherry & $\mathrm{y}=-0,077 \mathrm{x}^{2}+0,878 \mathrm{x}+90,18$ & $\mathrm{R}^{2}=0,98$ \\
Golden Yellow & $\mathrm{y}=-0,137 \mathrm{x}^{2}+1,193 \mathrm{x}+99,52$ & $\mathrm{R}^{2}=0,99$ \\
Salmon Rose & $\mathrm{y}=-0,035 \mathrm{x}^{2}-0,019 \mathrm{x}+87,04$ & $\mathrm{R}^{2}=0,99$ \\
Orange & &
\end{tabular}

$\mathrm{S} 1: \mathrm{CE}$ de 0,92 e $1,07 \mathrm{dS} \mathrm{m}^{-1}$; $\mathrm{S} 2: 1,76$ e 2,04 dS m $\mathrm{m}^{-1}$ (ambos no período vegetativo e reprodutivo respectivamente). 

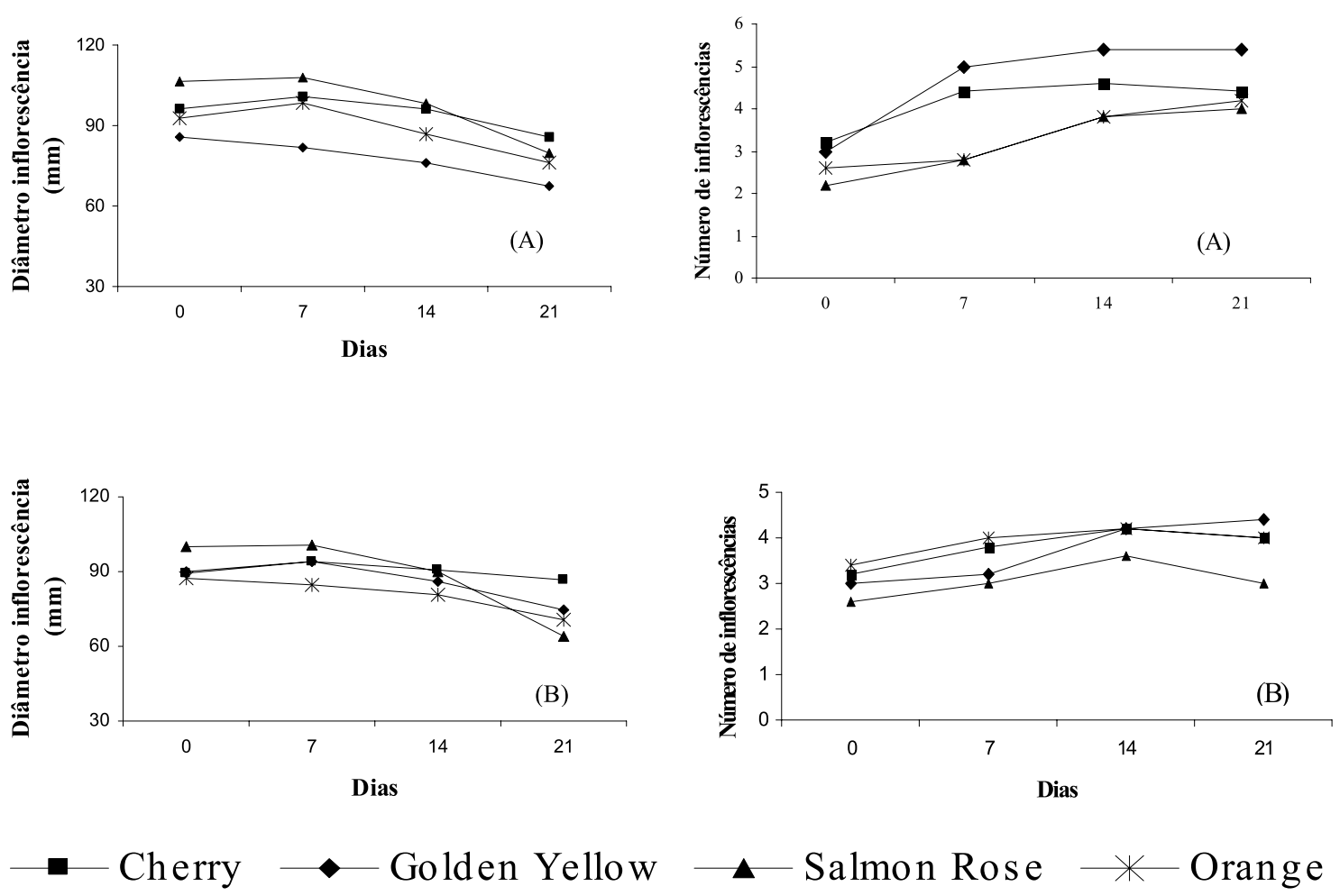

Figura 1. Diâmetro e número de inflorescências de quatro cultivares de gérbera de vaso fertirrigadas com duas soluções nutritivas, S1 (A) e S2 (B). Botucatu (SP), 2006.

Figure 1. Diameter and number for flower of four cultivar potted gerbera in two nutrient solutions, $S 1$ (A) and $S 2$ (B). Botucatu (SP), 2006. 